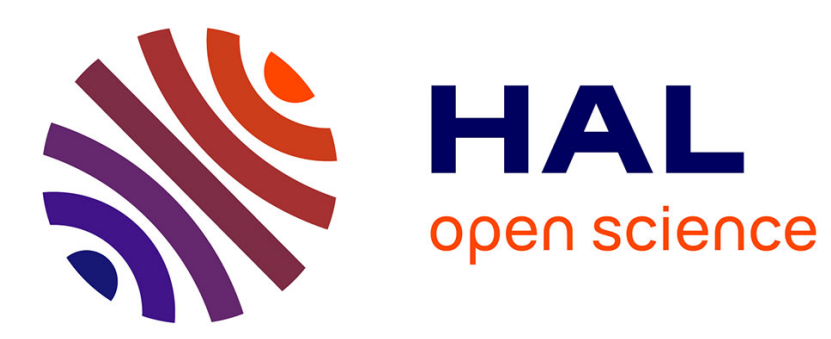

\title{
A BCMP extension to multiserver stations with concurrent classes of customers
}

Jean-Yves Le Boudec

\section{To cite this version:}

Jean-Yves Le Boudec. A BCMP extension to multiserver stations with concurrent classes of customers. [Research Report] RR-0390, INRIA. 1985. inria-00076166

\section{HAL Id: inria-00076166 \\ https://hal.inria.fr/inria-00076166}

Submitted on 24 May 2006

HAL is a multi-disciplinary open access archive for the deposit and dissemination of scientific research documents, whether they are published or not. The documents may come from teaching and research institutions in France or abroad, or from public or private research centers.
L'archive ouverte pluridisciplinaire HAL, est destinée au dépôt et à la diffusion de documents scientifiques de niveau recherche, publiés ou non, émanant des établissements d'enseignement et de recherche français ou étrangers, des laboratoires publics ou privés. 


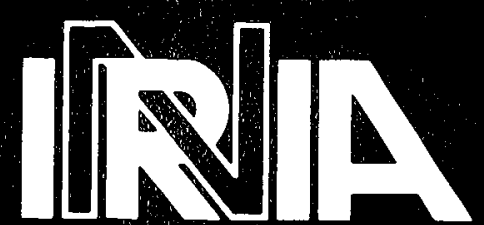

- CENTRE DE RENNES

I RISA

\section{Rapports de Recherche}

$\mathrm{N}^{\circ} 390$

\section{A BCMP EXTENSION TO MULTISERVER STATIONS WITH CONCURRENT CLASSES OF CUSTOMERS}

Jean-Yves LE BOUDEC 
Campus Universitaire de Beaulieu

Avenue du Général Leclerc 35042 - RENNES CEDEX FRANCE

Tél. : (99) 36.20.00

Télex : UNIFISA $950473 \mathrm{~F}$

A B CMP EXTENSION TO MULTISERVER STATIONS WITH CONCURRENT CLASSES OF CUSTOMERS

\author{
$\frac{\text { Publication Interne } n^{\circ} 250-\text { Mars } 85}{32 \text { pages }}$ \\ J.-Y. LE BOUDEC \\ INSA - IRISA
}

\title{
ABSTRACT
}

Consider a multiclass service station with B identical exponential servers, with constant rate $\mu$. At the station, the classes of customers are sorted into $M$ concurrency groups; the discipline of service is on a first come first served basis, but two customers of the same group cannot be served simultaneously. We show that product form is maintained when such stations are inserted in BCMP networks, and give closed form expressions for the steady-state probabilities.

\section{RESIME}

On considère une station de service multiclasse, possédant 8 serveurs exponentiels identiques, à taux constant. Dans la station, les classes de clients sont réparties en $M$ groupes de concurrence : deux clients dont les classes appartiennent au même groupe ne peuvent être servis simultanément. L'ordre d'attente est l'ordre d'arrivée à la file. On prouve que l'insertion de telles stations dans des réseaux B CMP conserve la forme produit, et on donne des formules explicites pour les probabilités stationnaires. 


\section{INTRODUCTION}

1 THE CONCURRENCY MULTISERVER STATION IN ISOLATION

1.1 Description of the model

1.2 Analysis of the states

1.3 The steady-state probabilities

1.4 Aggregation of results

1.5 The open case

2 INCLUSION OF CONCURRENCY MULTISERVER STATIONS IN B CMP NETWORKS

2.1 Closed form expression of the steady-state probabilities

2.2 Proof of theorem 5

2.3 Aggregation of results

CONCLUSION - Acknowledgement

APPENDIX : proof of the lemmas 
INTRODUCTION

BALBO, CHIOLA, DONATELLI and MARSAN study in [BCDM] a model of multiple bus multiprocessor system by using generalised stochastic Petri nets techniques. It is found that the local balance equation holds for this model, and a proof is given when the number of busses is 2 or 3 .

In this paper, we give an equivalent model, using queueing theory concepts:

In part 1 we study the system in isolation : we prove that local balance holds, and obtain closed form expressions for the steady state probabilities. In part 2 , we show that product-form still holds when we replace the classical FIFO exponential station with class independant rate of service in BCMP networks by an exponential station (still with class independant rates) with several servers and concurrent classes of customers. For that purpose, we examine local balance in detail, and use PELLAUMAIL and LE NY's technique to obtain product form.

\section{THE CONCURRENCY MULTISERVER STATION IN ISOLATION}

\section{I Description of the model}

We consider the following loop, consisting in two stations, which we call the inside and the outside stations :

- the inside station is a concurrency multiserver station ; namely :

* there are $B$ identical exponential servers (rate $\mu$ )

* the queueing disciplineis on a first come first served basis, but there is some concurrency in that two customers of the same class cannot be served simultaneously.

Hence a customer can be kept waiting in the inside station :

* either because all B servers are busy

* or because there already is a customer of the same class being served. 
In the sequel, ve shall say that a class is active if one customer of the class is receiving service.

When one or more server is available, the first customer belong ing to a non-active class enters the service center.

- the outside station is a one-class exponential station, with parameter $\lambda$.

- a customer leaving the outside station will belong to class $k$ with probability $q_{k}$, according to a Bernouilli trial. On leaving the inside station, a customer loses any information about its class.

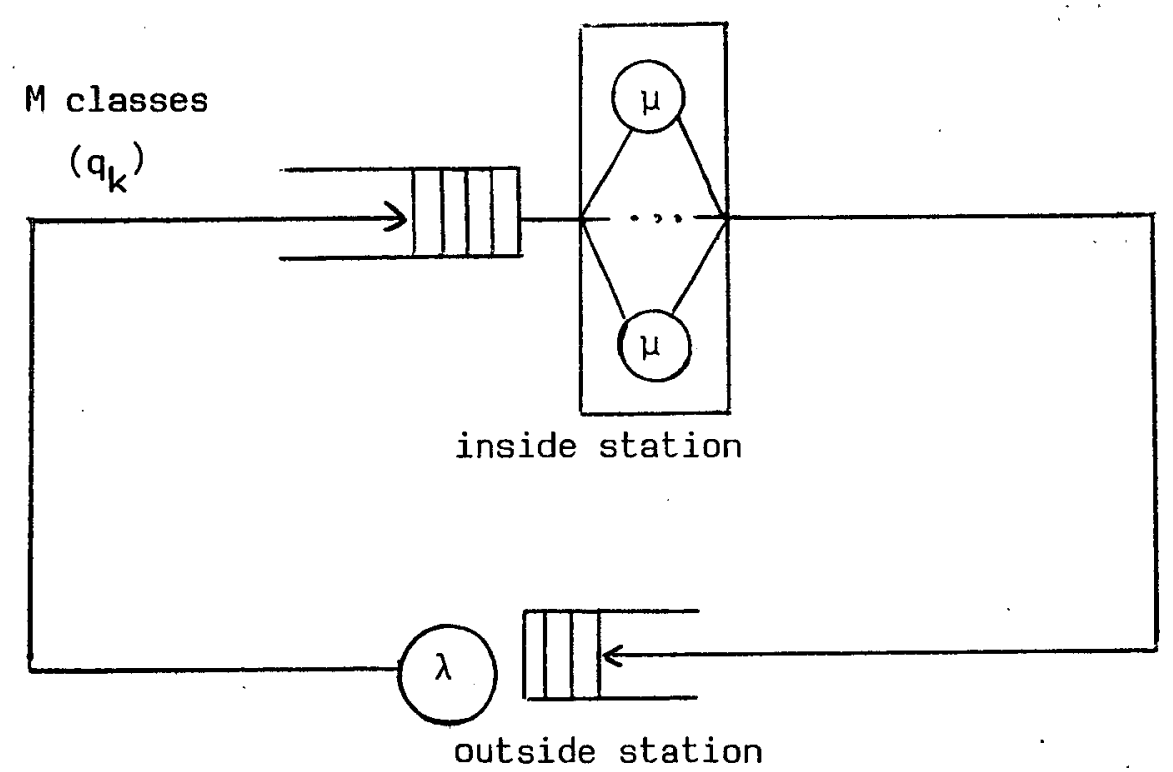

Like the model presented by BALBO et al. in [BCDM], the inside station represents parallel access to $M$ memory modules through $B$ busses (a customer belongs to class $k$ if it wants to have access to the $k^{\text {th }}$ module). The outside station is an auxiliary one, which will disappear in section 2.

We have assumed that the ammount of time spent in gaining access to a memory module is exponentially distributed, with a parameter that does not depend 
on the class of customer.

\section{Extension}

In section 2 we shall insert such stations in BCMP networks. For that purpose, it is preferable to consider a class-concurrency model : the set of all classes is divided into concurrency groups; and two customers of the same concurrency group cannot be served simultaneously. In this case, we shall also say that a group is active if one customer of a class

" belonging to the group is receiving service.

\subsection{Analysis of the states}

We use the following notations :

$A$ : the set of all active classes (or groups in the extended case). $\underline{c}=\left(c_{1}, \ldots, c_{S}\right)$ where $c_{i}$ is the class of the $i$ th customer vaiting. (in the inside station)

$N$ : the total number of customers in the loop.

Then a state of our system is described by :

$(A, \underline{c})$, or $A$ (empty waiting room), where :

- $A$ is a subset of at most $B$ classes 1 .. M

- $c$ is such that : if \#A<B then $c_{1}, \ldots, c_{S} \in A$

$$
\text { else } c_{1}, \ldots, c_{S} \in\{1, \ldots, M\}
$$

- \#A+s\&N (s is the size of sequence $\underline{c}$ )

$\emptyset$ is the state where the inside station is empty.

Now we have to introduce a notion that will be necessary to obtain the steady-state probabilities :

For every position $i$ in the waiting room, and for a given set of active classes, we define level $N(i)$ by :

- if there are only active-class-customers waiting in positions 1 to $i$, 
then $N(i)$ is the number of those active classes (i.e., the number of active classes that are represented in positions 1 to $i$ of the waiting room).

- if there is (at least) one customer of non active class in positions 1 to $i$, then $N(i)=B$ (the number of servers).

- as a convention, we also write $N(0)=0$.

We give examples when the number of servers is 3 :

Examples $\quad(B=3)$

$\underline{\operatorname{ex} 1}$

\begin{tabular}{|c|c|c|c|c|}
\hline state & $\{2,4,3\}$ & 33 & 433 & 24123 \\
\hline levels & $\begin{array}{l}\text { service } \\
\text { center }\end{array}$ & 1 & 2 & $3=B$ \\
\hline
\end{tabular}

$\underline{\operatorname{ex} 2}$

\begin{tabular}{l|c|c|cc} 
state & $2,4,3$ & 33 & 13324 & 32423 \\
\hline levels & $\begin{array}{c}\text { service } \\
\text { center }\end{array}$ & $1 \underbrace{1}_{\text {waiting room }}$
\end{tabular}

ex 3

\begin{tabular}{l|c|c|c} 
state & 2,3 & 33 & 2333 \\
\hline levels & $\begin{array}{c}\text { service } \\
\text { center }\end{array}$ & $\underbrace{1}_{\text {waiting room }}$ & 2
\end{tabular}.

In the extended case, the phrase "active class" has to be replaced by "active group of classes". We also give an example in that case :

\section{Example $\quad(B=3)$}

Set of classes $=\{1,2,3,11,12,21,22,31\}$

concurrency groups : $\{1,2,3\}\{11,12\}\{21,22\}\{31\}$ 
$\underline{\operatorname{ex} 4}$

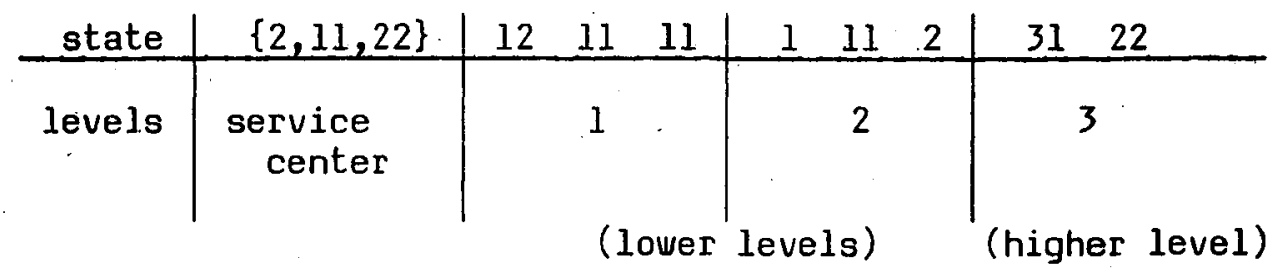

(In the sequel, we shall refer to levels 1 to $B-1$ as the lower levels; the number of lower levels is possibly less than B-1).

\subsection{The steady state probabilities}

Note $\rho_{k}=\frac{\lambda}{\mu} q_{k} \quad$ for all class $k$. Then :

Theorem 1

The steady-state probability of the system described in $1-1$ is given, for every feasible state, by :

$p\left(\left\{b_{1}, \ldots, b_{R}\right\}, c_{1}, \ldots, c_{s}\right)=k\left(\prod_{i=1}^{R} \rho_{b_{i}}\right)\left(\prod_{i=1}^{s} \frac{\rho_{c_{i}}}{N(i)}\right)$

where $k$ is a normalising constant, and level $N(i)$ is defined in section $1-2$.

(In the formula, $b_{1} \ldots b_{R}$ are the $R \leqslant B$ active classes; feasible states are defined in $1-2$ ).

Proof. It is clear that any feasible state of the loop can be reached from any other feasible state, which proves ergodicity, as there are only finitely many states.

Now to prove theorem 1 it is sufficient to show that (1-2) checks the balance equations; indeed, more detailed equations are satisfied : 
(detailed equations 1)

departure rate from state $(A, \underline{c})$ through a departure from the inside station

(1-3) = arrival rate at state $(A, \underline{C})$ through an arrival at the inside station.

(detailed equations 2)

"local balance at the inside station"

departure rate from state $(A, \underline{C})$ through a class-k-customerarrival at the inside station

(1-4) = arrival rate at state $(A, C)$ through a class-k-customerdeparture from the inside station.

It is clear that if the first set of equations, and the second set for all $k$, are checked, then the Chapman-Kolmogorov equations also are. For exact relations to what is usually called local balance, see section 2 . Let us now examine the detailed equations ; first, we assume each concurrency group is reduced to one class.

a) detailed equations 1

We have to distinguish several cases, according to the states of the vaiting room.

case al the higher level is not empty (hence all B servers are busy). Eq 1-3 now writes :

$$
\begin{aligned}
& p(A, \underline{c}) B \mu=p\left(A, \underline{c}-c_{s}\right) \lambda q_{c_{s}} \text { where } c_{s} \text { is the last element of } \\
& \text { sequence } c \text {, and } \underline{c}-c_{s} \text { is short for }\left(c_{1}, c_{2}, \ldots, c_{s-1}\right)
\end{aligned}
$$

case_a2 the higher level is empty but the waiting room is not.

In this case, we call :

- R : the number of busy servers 
- F : the set of the classes that are represented in the waiting room. As the $B-t h$ level is empty, $F$ is necessarily a subset of $A$.

With these notations, equation 1-3 writes :

$(1-6)$

$$
p(A, \underline{c}) k \mu=p\left(A, \underline{c}-c_{s}\right) \lambda q_{c_{s}}+\sum_{k \in A-F} p(A-\{k\}, \underline{c}) \lambda q_{k}
$$

(As usual, the last sum is 0 if $A=F$ ).

case a3 the waiting room is empty; we have :

$(1-7)$

$$
p(A) R \mu=\sum_{k \in A} p(A-\{k\}) \lambda q_{k}
$$

In all three cases it is straightforward to prove that the equations are satisfied. Indeed, it is eqs (1-5) to (1-7) that lead to the closed form expression of $p$ in theorem 1 .

b) "local balance at the inside station"

In this section, class $k$ and state $(A, \underline{c})$ are fixed. All implicit references are to state $(A, \underline{c})$. We also distinguish several cases, according to the state of the service center. We assume that the outside station is not empty $(\# A+s<N-1)$ or else eq $1-4$ is trivial.

case bl $k$ is not an active class $(k \notin A)$ and all servers are busy $(\mathrm{R}=\mathrm{B})$. Eq $1-4$ writes :

$$
p(A, \underline{c}) \lambda q_{k}=\sum_{\substack{b \in A \\ 0 \leqslant \ell \leqslant D(b)}} p\left(A_{b-}, k^{+}, c_{-b^{+}}^{\ell}\right) \mu
$$

with the following notations :

$-A_{b-, k^{+}}=(A-\{b\}) \cup\{k\}$

- $\mathrm{c}_{\mathrm{b}}^{\ell}$ is the state of the vaiting room obtained after adding a class $b$ customer just after the $\ell^{\text {th }}$ position. 
- For all active class $b, D(b)$ is defined by :

- $1+D(b)$ is the first position $i$ of the waiting room where the following condition is true : "position $i$ belongs to the higher level or the customer vaiting in position $i$ belongs to class $b$ ".

- if the vaiting-room is empty, then $D(b)=0$;

- if there are no class $b$ customers and no higher level, then $D(b)$ is the last position of the waiting room.

For instance, with the examples of section 1-2, we have :

\begin{tabular}{c|c|c|c} 
example & $D(2)$ & $D(3)$ & $D(4)$ \\
\hline 1 & 5 & 0 & 2 \\
\hline 2 & 5 & 0 & 2 \\
\hline 3 & 2 & 0 &
\end{tabular}

We shall now check the equation :

when state $(A, \underline{c})$ is changed to state $\left(A_{b-}, k^{+}, c_{b^{+}}^{l}\right)$, then all positions in the vaiting room from $\ell$ to $s$ (the last one), move to level $B$, since class $k$ is inactive in the new state. Hence :

$$
p\left(A_{b^{-}, k^{+}}, \underline{c}_{b^{+}}^{\ell}\right)=p(A, c) \times o_{k} \times \frac{1}{B} \times{\underset{l}{\ell^{\prime}=\ell+1}}_{\Pi}^{s} \frac{N\left(\ell^{\prime}\right)}{B}
$$

the proof now ends with the use of lemma 1 (see appendix), which states that :

Lemma 1 $\sum_{\substack{b^{L} \in A \\ 0 \leqslant \ell \leqslant D(b)}} \frac{1}{B} \prod_{\ell^{\prime}=\ell+1}^{s} \frac{N\left(\ell^{\prime}\right)}{B}=1$

case b2 $k$ is not an active class, and there are $R<B$ busy servers ; with the same notations as in bl, the equation writes : 


$$
p(A, \underline{c}) \lambda q_{k}=p(A \cup\{k\}, \underline{c}) \mu
$$

In that case the proof is obvious.

case_b3 $k$ is an active class; the equation is :

(1-10) $\quad p(A, \underline{c}) \lambda q_{k}=\sum_{0<\ell \leqslant D(k)} p\left(A, \underline{c}_{k^{+}}^{\ell}\right) \mu$

To prove that eq $1-10$ is satisfied, first notice that when adding a class $k$ customer after the $\ell^{\text {th }}$ position, the levels of all positions from $(l+1)$ to $D(k)$ increase by one ; hence :

$$
p\left(A, \underline{c}_{k^{+}}^{\ell}\right)=p(A, \underline{c}) \rho_{k} \frac{1}{N(\ell)+1} \underbrace{D(k)}_{l^{\prime}=\ell+1} \frac{N\left(\ell^{\prime}\right)}{N\left(\ell^{\prime}\right)+1} \quad 0<\ell<D(k)
$$

Now eq $1-10$ is satisfied thanks to lemma 2, the proof of which is given in appendix :

Lemma 2

$$
\sum_{0<\ell \leqslant D(k)} \frac{1}{N(l)+1} \prod_{\ell^{\prime}=\ell+1}^{D(k)} \frac{N\left(\ell^{\prime}\right)}{N\left(\ell^{\prime}\right)+1}=1
$$

In a second step, we have to extend the proof to the case of concurrency groups. There is no difficulty in this :

- in part a), just replace "active class" by "active group"

- in part b), in the definition of $D(b)$, replace condition "the customer waiting in position $i$ belongs to class $b$ " by "the customer waiting in position $i$ 'belongs to the concurrency group of class $b "$.

(We could also notice the following : aggregate every concurrency group into one class, then the repartition of classes inside a given concurrency group is independant of the aggregated state of the loop). 


\section{1-4 Aggregation of results}

In this section ve compute :

$$
\begin{aligned}
P\left(A, n_{1}, \ldots, n_{M}\right)=\operatorname{Pr}\{ & \text { the set of all active classes is } A, \\
& \text { and there are } n_{c} \text { class } c \text { customers in } \\
& \text { the waiting-room, for all } c=1 \ldots M\} .
\end{aligned}
$$

We first consider the restricted case (one class per group);

without loss of generality, we can suppose $A=\{1, \ldots, R\},(R<B)$.

$$
\begin{aligned}
& \text { Theorem } 3 \quad \text { a) } P\left(\{1, \ldots R\}, n_{1}, \ldots, n_{R}, 0 \ldots 0\right)=\prod_{k=1}^{R} \rho_{k}^{n_{k}^{+1}} p(\emptyset) \quad(R \leqslant B) \\
& \text { b) } P\left(\{1, \ldots . B\}, n_{1}, \ldots, n_{B}, n_{B+1}, \ldots, n_{M}\right) \\
& =\prod_{k=1}^{B} \rho_{k}^{n_{k}+1} \prod_{k=B+1}^{M} \rho_{k}^{n_{k}} \frac{1}{B|\vec{n}|} \pi(\vec{n}) p(\emptyset) \\
& \text { (1-11) } \\
& \text { where } \pi(\vec{n}) \text { is recursevely defined by : } \\
& \left\{\begin{array}{l}
\pi(\vec{n})=B^{|\vec{n}|} \quad \text { if } \quad\left(n_{B+1} \cdots n_{M}\right)=\overrightarrow{0} \\
\pi(\overrightarrow{0})=1 \\
\pi(\vec{n})=\sum_{k / n_{k}>0} \pi\left(\vec{n}_{k^{-}}\right) \quad \text { else }
\end{array}\right. \\
& \text { and }|\vec{n}|=\sum_{i=1}^{M} n_{i}
\end{aligned}
$$

Note that a) is a particular case of $b$ since if $R<B$ then $n_{k}=0$ for all $k>R$.

Proof a) the idea of the proof is simple : if the number of classes is at most $B$, then the inside station is equivalent to $B=M$ separate FIFO queues.

Formally, for $\vec{m}$ in $\mathbb{N}^{M}$, call : 
$E_{n}$ : the set of all possible states with $m_{k}$ class $-k$ customers, for all $k$. Then :

$(1-33)$

$$
\begin{aligned}
& P\left(\{1, \ldots, R\}, n_{1}, \ldots, n_{R}, 0, \ldots, 0\right)=\sum_{(A, \underline{c}) \in E_{n^{\prime}} p(A, \underline{c})} p \\
& \text { with } \vec{n}^{\prime}=\left(n_{1}+1, \ldots, n_{R^{\prime}}+1,0, \ldots, 0\right) .
\end{aligned}
$$

Summing eq $1-6$ over all states in $E_{n^{\prime}}$, we obtain :

$$
\begin{aligned}
P\left(\{1 \ldots R\}, n_{1} \ldots n_{R}, 0 \ldots 0\right) R \mu & =\sum_{k / n_{k}>0} \sum_{c \in C_{k}} p(\{1 \ldots R\}, \underline{c}-k) \lambda q_{k} \\
& +\sum_{1 \leqslant k \leqslant R / n_{k}=0} p(\{1 \ldots R\}-\{k\}, \underline{c}) \lambda q_{k}
\end{aligned}
$$

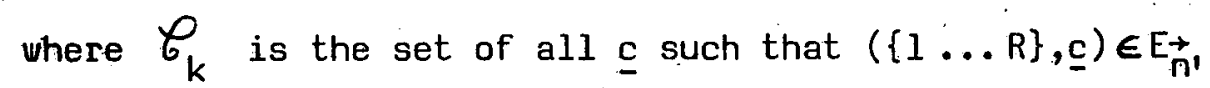
and whose last element is $k$; note that the set of all $(\{1 \ldots R\}, c-k)$ when $c$ is in $\mathscr{C}_{k}$ is exactly $E_{n_{k-}} \quad\left(\right.$ if $\left.n_{k^{\prime}}>0\right)$.

Hence ve can write :

(1-14) $\quad P^{\prime}\left(\vec{n}^{\prime}\right) R \mu=\sum_{k / n_{k}^{\prime}>0} P^{\prime}\left(\vec{n}_{k-1}^{\prime}\right) \lambda q_{k}$

where $P^{\prime}\left(\vec{n}^{\prime}\right)=P(\{1, \ldots, R\}, \vec{n})$

and $P^{\prime}\left(\vec{n}_{k_{-}}\right)=P\left(\{1, \ldots, R\}, \vec{n}_{k^{-}}\right)$if $n_{k}>0$

$$
=P(\{1, \ldots, R\}-\{k\} ; \vec{n}) \text { if } n_{k}=0 \text { and } 1 \leqslant k \leqslant R \text {. }
$$

Recursion 1-14 easily results in :

$p \cdot\left(\vec{n}^{\prime}\right)=\rho_{1}^{n^{\prime}} 1 \ldots \rho_{R}^{n_{R}^{\prime}} p(\emptyset)$.

b) Call $\mathrm{F}_{\mathrm{m}}$ the set of all $\underline{c}$ for which the total number of class $k$ customers is $m_{k}$, for all $k$.

If $\underline{c} \in F_{\vec{m}}$ and if $m_{k}>0$ for at least one $k>B$, then the higher level of $\subseteq$ is not empty; hence, summing $1-5$ over 
all $\underline{c}$ in $F_{\vec{m}}$, we obtain :

$$
P(\{1 \ldots B\}, \vec{n}) B \mu=\sum_{k / n_{k}>0} \sum_{\underline{\epsilon} \in F_{\vec{n}}} \underset{p(\{1 \ldots B\}, c-k)}{p} \lambda q_{k}
$$

and then

$$
P(\{1 \ldots B\}, \vec{n}) B \mu=\sum_{k / n_{k}>0} P\left(\{1 \ldots B\}, \vec{n}_{k^{-}}\right) \lambda q_{k}
$$

(for all $\vec{n}$ such that $\left(\vec{n}_{B+1} \ldots \vec{n}_{M}\right) \neq \overrightarrow{0}$ )

let $P(\{1 \ldots B\}, \vec{n})=\frac{1}{B|\vec{n}|} \prod_{k=1}^{M} \rho_{k}^{n_{k}} \times\left(\prod_{k=1}^{B} \rho_{k}\right) \times \pi(\vec{n})$

then $1-15$ gives

$\pi(\vec{n})=k t \cdot q \cdot n_{k}>0 \pi\left(\vec{n}_{k}\right)$

If $n_{B+1}=\ldots=n_{M}=0$, then part a) gives $\pi(\vec{n})=B^{\mid \vec{n}} \mid$, which ends the proof of theorem 3 .

\section{Extended case}

In this case we note l...M the groups of classes; a class of customer is written $(m, c)$ where $m$ is the group it belongs to. Then theorem 3 still holds if $\{1 \ldots R\}^{*}$ is the set of active groups, $\cdot \vec{n}_{m}$ is the number of customers in the waiting room belonging to group $m$, and $\rho_{m}=\sum_{c} \rho_{(m, c)}$.

In section 2, we shall be interested in getting the repartitions inside each group of classes. First we shall make our notations more precise :

- $c_{m}$ is the number of classes in group $m$

- $n_{m, k}$ is the number of class $-(m, k)$ customers $1 \leqslant k \leqslant c_{m}$ and $\vec{n}_{m}=\left(n_{m, 1}, \ldots, n_{m, c}\right)$

$\vec{n}_{m}=\left|\vec{n}_{m}\right|=$ the number of group-m customers. 
$-\bar{P}\left(\left\{\left(1, i_{1}\right) \ldots\left(R, i_{R}\right)\right\} \vec{n}_{1}, \ldots, \vec{n}_{M}\right)$ is the steady-state probability that the active classes are $\left(1, i_{1}\right) \ldots\left(R, i_{R}\right) \quad$ [hence the active groups are $1 \ldots R$ ] and that the number of class $(m, k)$ customers in the waiting room is $n_{m, k}$.

Using the conditional independance of classes inside a group, and theorem 3, we easily obtain :

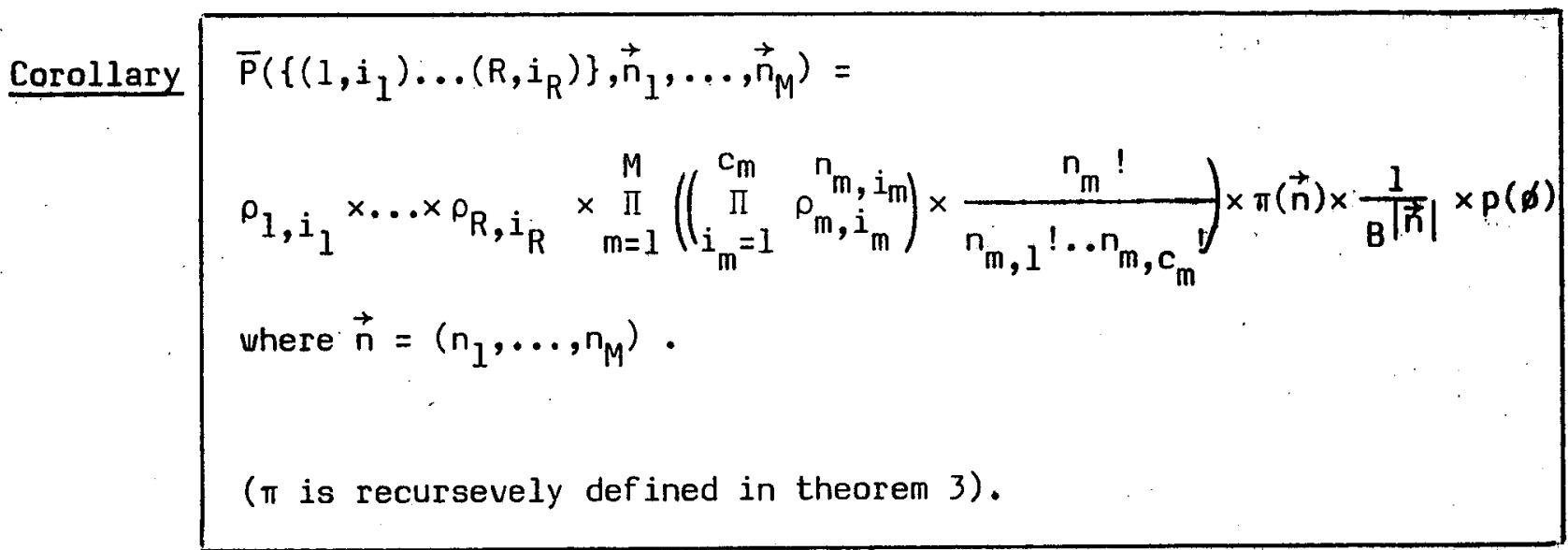

\section{1-5 The open case}

We now consider the case where the initial number of customers in the inside station is infinite; this system is equivalent to the concurrency multiserver station, alone, fed by a Poisson process :

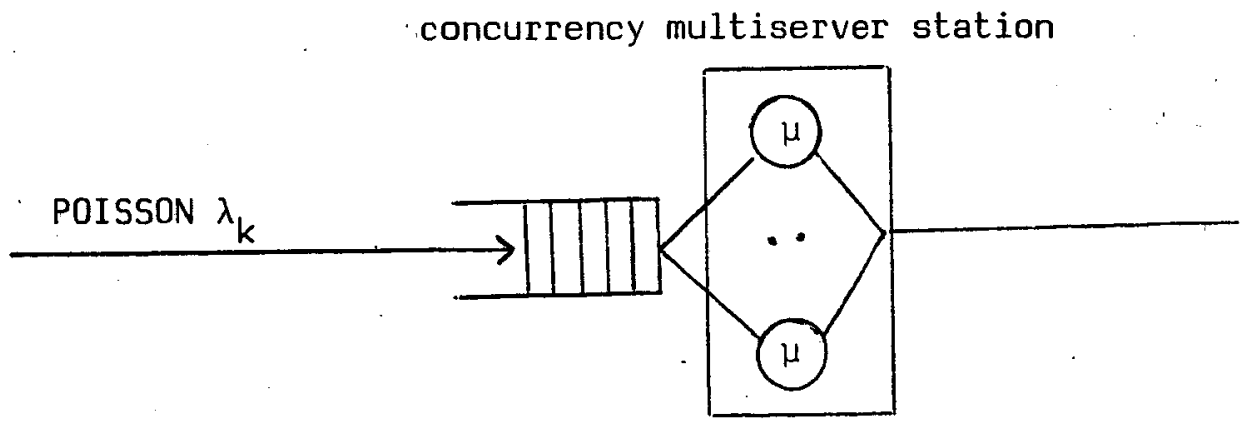


We note $\lambda_{k}$ the arrival rate for class-k customers, and we consider the restricted case (one class per concurrency group). Then the only difference with the preceeding sections concerns stability :

\section{Theorem 4}

$(1-16)$

$(1-17)$
1) The open system is ergodic if and only if :

(i) for all class $k, \lambda_{k}<\mu$

(ii) $\sum_{k=1}^{M} \lambda_{k}<B \mu$

2) If the system is ergodic, the steady-state probability for every feasible state is given by ( $1-2$ ) ; aggregation formulae ( $1-11)$ also hold.

Proof It is clear that $p(A, \underline{C})$, as given in part 2$)$ is a solution of the balance equations.

a) Now suppose conditions ( $i$ ) and (ii) hold; since the set of all feasible sets is irreducible, in order to prove that the system is ergodic, it is sufficient to prove that :

$$
\sum \mathrm{p}(\mathrm{A}, \underline{\mathrm{c}})<+\infty
$$

$(A, \underline{c})$ feasible

Using aggregation formulas $(1-11)$, that is equivalent to :

$(1-18)$ $\sum \pi_{A}(\vec{n}) \prod_{i=1}^{M}\left(\frac{\rho_{i}}{B}\right)^{n_{i}}<+\infty$, for all set of active classes $A$ $\vec{n}$ feasible

- If \#A<B, then $\pi_{A}(\vec{n})=B|\vec{n}|$ and the set of feasible $\vec{n}$ is $\left\{\vec{n} \in \mathbb{N}^{M} / n_{i}=0\right.$ if $\left.i \notin A\right\}$; hence the sum in $(1-18)$ becomes :

$(1-19)$

$$
\prod_{i \in A}\left(\sum_{i} \rho_{i} \rho_{i}\right)
$$


which is finite because all $\rho_{i}$ are less than 1 .

- Now let $\# A=B$; we can suppose $A=\{1, \ldots B\}$; let $\vec{X}=\left(x_{1} \ldots x_{B}\right), \vec{Y}=\left(x_{B+1} \ldots x_{M}\right)$, and :

$F_{N}(\vec{X}, \vec{Y})=\sum_{\vec{n} / n_{1}+\ldots+n_{M}=N} \pi(\vec{n}) x_{1}^{n_{1}} \ldots x_{M}^{n_{M}}$

Then recursion ( $1-12$ ) yields

$(1-20)$

$F_{N}(X, Y)-F_{N}(X, 0)=\left(\sum_{i=1}^{M} X_{i}\right) F_{N-1}(X, Y)-\left(\sum_{i=1}^{B} X_{i}\right) F_{N-1}(X, 0)$

Writing (1-20) for all $n=1$ to $N$, we obtain :

$F_{N}(X, Y)=x^{N}-x^{\prime} \sum_{n=0}^{N-1} x^{n} F_{N-1-n}(X, 0)+\sum_{n=0}^{N-1} x^{n} F_{N-n}(X, 0)$

where: $x=\sum_{i=1}^{M} x_{i}, \quad x^{\prime}=\sum_{i=1}^{B} x_{i}$ and $F_{0}(x, y)=1$

which leads to :

(1-21) $\quad F_{N}(X, Y)=\left(x-x^{\prime}\right) \sum_{k=0}^{N-1} x^{k} F_{N-1-k}(X, 0)+F_{N}(x, 0)$

Now let $x_{i}=\frac{\rho_{i}}{B}$ in $1-21$; summing over all $N$, we have :

(1-22) $\quad \sum_{N} F_{N}(X, Y)=\left(x-x^{\prime}\right)\left(\sum_{k \geqslant 0} x^{k}\right)\left(\sum_{N \geqslant 0} F_{N}(x, 0)\right)+\sum_{N \geqslant 0} \cdot F_{N}(x, 0)$

now $\sum_{N} F_{N}(X, 0)=\sum_{n_{1}+\ldots+n_{B}}=n \quad \prod_{i=1}^{B} \rho_{i}^{n_{i}}<+\infty$ since $\rho_{i}<1$ for all $i$

and $x^{\prime}<x<1 \quad$ since $\sum_{i=1}^{M} \rho_{i}<B$;

hence $1-18$ is proved for $A=\{1, \ldots, B\}$.

b) conversely, if $\rho_{i} \geqslant 1$ for some $i$, or $\sum_{i=1}^{M} \rho_{i} \geqslant B$, then using $1-18,1-19$ or $1-22$, it is easy to prove that 
$(1-23)$

$$
\sum_{(A, \underline{c}) \text { feasible }} p(A, \underline{c})=+\infty
$$

That proves that the Markov process that describes the open system is not ergodic, since if it were so, the Chapman-Kolomogorov equations vould have only one positive solution, and p obviously is.

Equations $1-19$ and $1-22$ also give the following result :

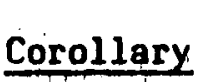

For all subset $A$ of $\{1, \ldots, M\}$, define $f(A)$ by :

- if $\# A<B$ then $f(A)=\prod_{i \in A} \frac{1}{1-\rho_{i}}$

- if $\# A \geqslant B$ then $f(A)=\left(\prod_{i \in A} \frac{1}{1-\rho_{i}}\right) \times \frac{1-\sum_{i \in A} \rho_{i}}{1-\rho}$

Then, if the queueing system is ergodic :

- the normalizing constant $K$ is given by :

$$
K^{-1}=\sum_{A \subset\{1 \ldots M\}} f(A)
$$

- the steady-state probability that the set of all active classes is $A$, is $K f(A)$. 
2 INCLUSION OF CONCURRENCY MULTISERVER STATIONS IN A BCMP NETWORK

\subsection{Closed form expression of the steady-state probabilities}

We consider a closed mult iclass network of queues, in which the service stations are

- either of the BCMP types

- or "concurrency multiserver" stations.'

Stations of the last type are studied in part 1 ; we recall here the properties of these stations :

- classes are gathered into groups ; at a given station, we shall note $(m, l)$ the $\ell^{\text {th }}$ class of the $m^{\text {th }}$ group.

- The queueing disciplineis on a first come first served basis, but two customers whose classes belong to the same group cannot be served simultaneously.

- All servers are identical and exponential, with constant rates.

Such stations are of some interest only if the number of groups (say $M$ ) is greater than the number of servers (say B). In that case, a customer can be kept waiting either because there is no server available, or because some customer of the same concurrency group is being served.

These stations can model parallel access to $M$ identical memory modules through $B$ busses : product form in such models was brought by to light BALBO - CHIOLA - DONATELL I - MARSAN.

Now we go further into the description of our network with some notations :

$-q_{i, c, i}{ }^{\prime}, c^{\prime}$ is the (routing-) probability, for a customer leaving station $i$ with class $c$ to enter station $i$ ' with class $c^{\prime}$; we assume $q_{i, c, i^{\prime}, c^{\prime}}=0$.

- $S$ is the number of stations and a state of the network is $\left(\vec{x}_{1}, \vec{x}_{2}, \ldots, \vec{x}_{S}\right)$ where $\vec{x}_{i}$ is an appropriate vector, depending on the type of the station : 
- if station $i$ is a concurrency-multiserver station, then $\vec{x}_{i}=\left(A_{i}, c_{i}\right)$ where $A_{i}$ is the set of the classes of the customers that are being served (active classes), and $\tau_{i}$ is the sequence of the classes of the customers that stand in the waiting-room, in FCFS order. Feasible states are defined in formulas 1-1. That still holds if station $i$ is a (BCMP-) FCFS exponential station, with class independant rate; in that case $A_{i}$ is the class of the customer being served, (if there is any, else $\emptyset$ ).

- else $\vec{x}_{i}=\left(\left(k_{i, 1}, s_{i, 1}\right) \ldots\left(k_{i, k_{i}}, s_{i, k_{i}}\right)\right)$ where $k_{i j}$ is the class of the $j$ th customer in the order of the station (LCFS or F(FS), and $s_{i, j}$ is the stage in the Cox representation.

- At a concurrency multiserver station, we also define some levels in the waiting-room :

given that the number of servers is $B_{i}$, and that the set of active classes is $A_{i}$, we define $N_{i, A_{i}}(j)$ for every position $j$ by :

- if all customers waiting in positions $1 \ldots j$ belong to active groups, the $N_{i, A_{i}}(j)$ is the number of active groups represented in positions $1 \ldots j$.

- if at least one of the customers waiting in positions $1 \ldots j$ belongs to an inactive group, then $N_{i, A_{i}}(j)=B_{i}$. (See section 1-2 for more details).

$-\left(e_{i, c}\right)_{i, c}$ is a positive solution of : $e_{i, c}=\sum_{j, d} e_{j, d} q_{j, d, i, c}$

- if station $i$ is of concurrency multiserver type, with rate $\mu_{i}$

for each server, then : $\rho_{i, c}=\frac{e_{i, c}}{\mu_{i}}$ for all class $c$.

We assume the network to be ergodic ; under this assumption, we have the following theorem : 


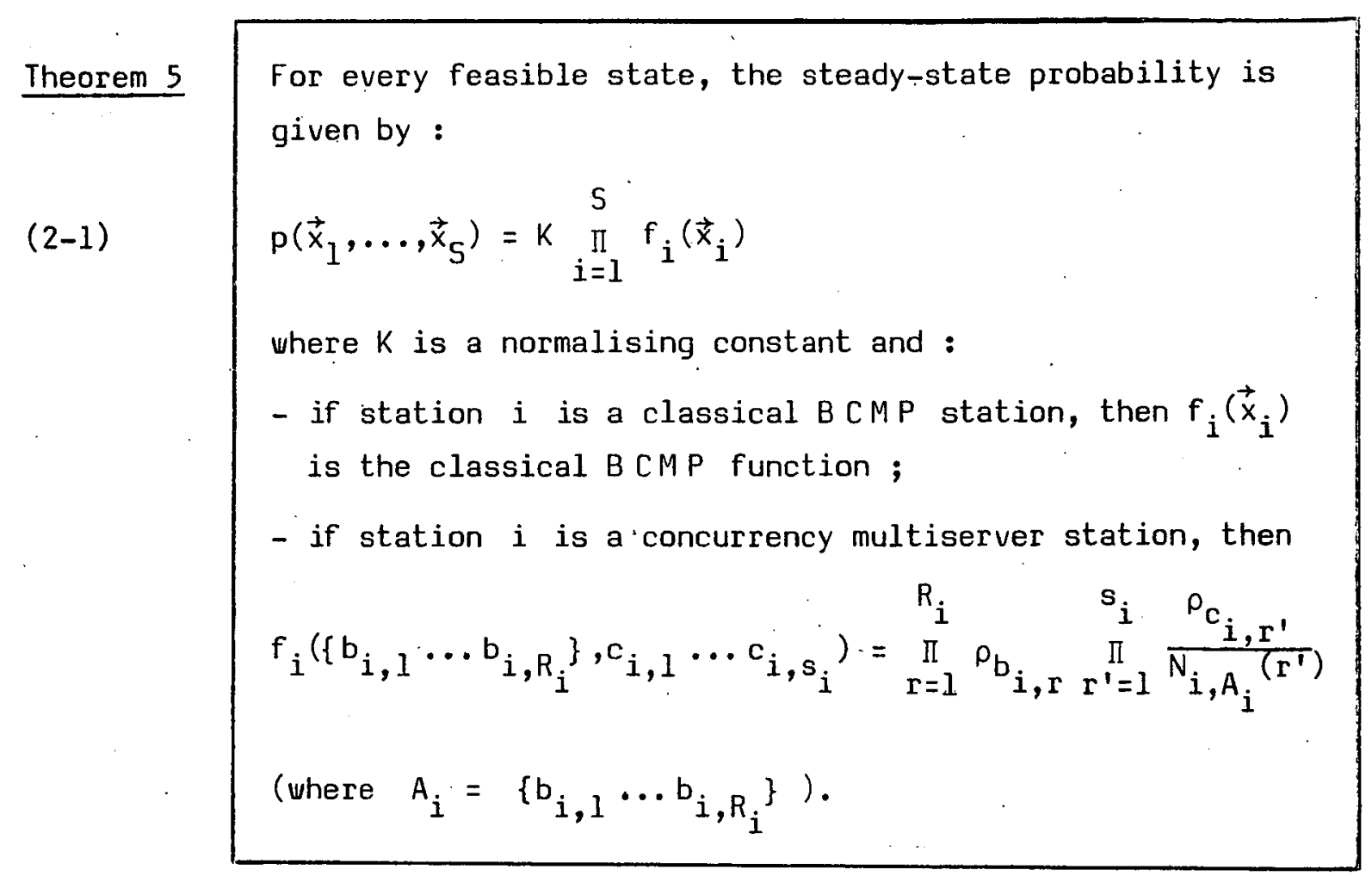

\subsection{Proof of theorem 5}

To prove theorem 5, we use the following property :

(2-2) If each station in isolation satisfies local balance, then the steady-state probability of the network has product form. ([Mun], [C.H.T.]).

We have to define what we call "local balance", since that phrase has been used with various meanings : this is done in a). A formal statement, equivalent to $2-2$, is given in $b$ ). Before going any further into the demonstration, notice that the network of section 2-1 is not locally balanced, i.e. local balance holds for every station in isolation, but not necessarily in the network.

\section{a) local balance in isolation}

Consider a multiclass station 5.5 is very general, it can be a closed markovian network, with transition rates that depend on the states of $S$ only. (We omit any formal definition). 
We connect $\mathrm{S}$ to an auxiliary one-class exponential station $\mathrm{S}_{0}$, with FIFO discipline and $\lambda=1$ service rate. The loop we obtain can be called : station $S$ in isolation.

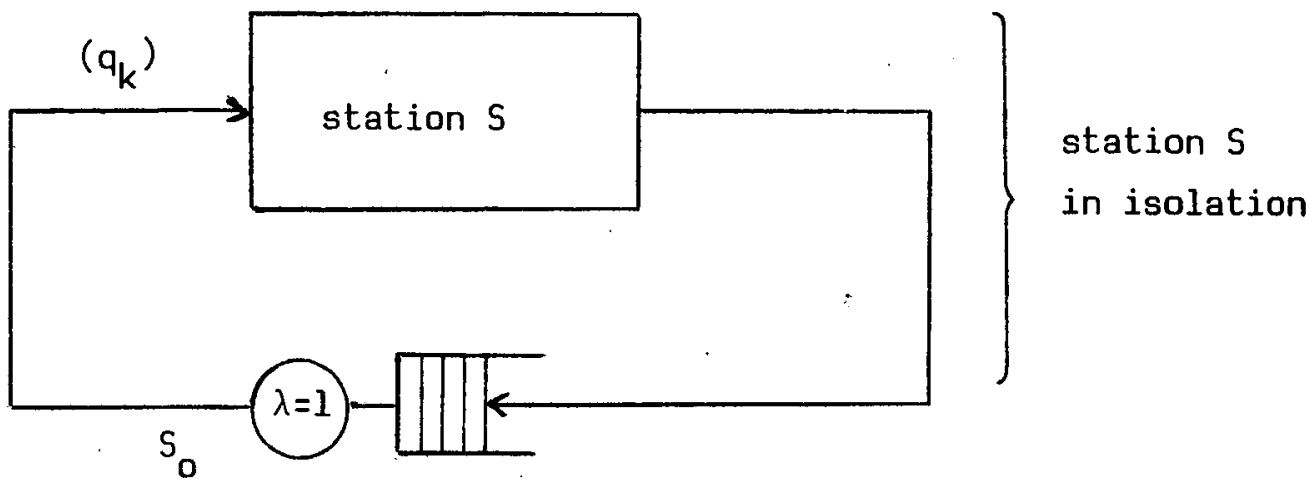

In the loop, a customer leaving station $S_{0}$ arrives at station $S$ in class $k$ with probability $q_{k}$; the total number of customers is $N$.

We say that local balance holds if the following set of equations is satisfied by the steady-state probabilities :

\section{Local balance}

for all class $k$, and all state e of the loop :

departure rate from state e through an arrival of a class $k$

$(2-3)$ customer at station $\mathrm{S}$

= arrival rate at state e through a departure of a class $k$ customer from station 5 .

Now we say that station S satisfies "local balance in isolation" if eqs 2-3 hold for any total number of customers in loop $R$, and any $q_{k}$. That is equivalent to the existence of functions $f$, 
depending 'on $q_{k}$, but not on $N$, such that :

$p_{N}(e)=c_{N} f(e)$ where $p_{N}$ is the steady-state probability of loop $R$ with $N$ customers, and $c_{N}$ is a normalizing constant.

Due to global balance in the loop, if eqs 2-3 hold, then the following system is satisfied :

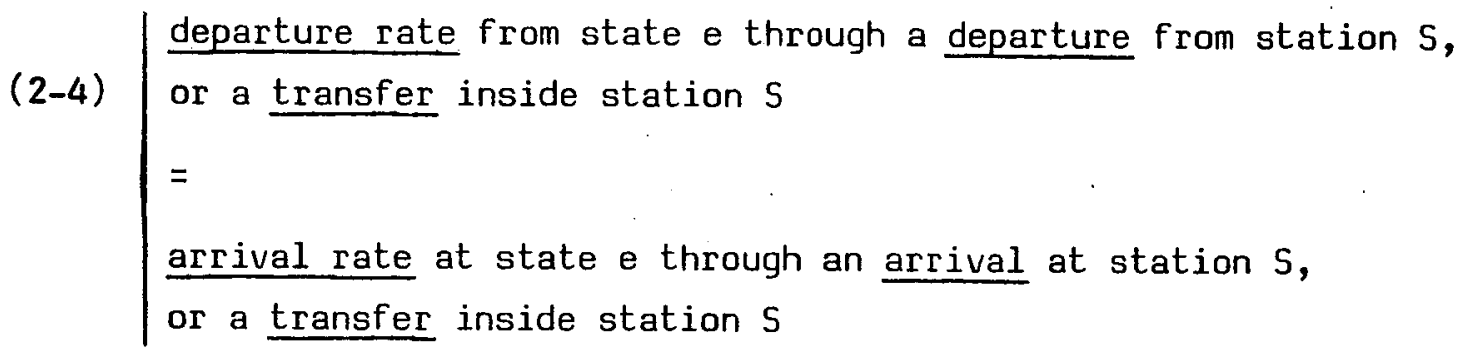

It is not required (and generally not true) that eq. 2-4 holds by class. If $S$ is a network of sub-stations, eq. 2-4 involves departures fromand arrivals at all sub-stations : there is no need for local balance inside $s$.

It is easy to prove that BCMP stations satisfy "local balance in isolation"; as for concurrency multiserver stations, the result is shown in section 1-3. Now to obtain formula 2-1 and a formal proof of 2-2, we use theorem 6, shown in the following section.

\section{b) Networks made of stations that are locally balanced in isolation}

\section{Theorem 6}

Consider a closed network $R$ made of $I$ stations that satisfy "local balance in isolation" (see 2-3). It is assumed to be ergodic.

For every station $s_{i}$, note $f_{i}\left(\vec{x}_{i}\right)$ the steady-state probability of the associated loop when $q_{k}=e_{i, k} \cdots$

Then for all feasible states of the network, the steady-state probability is given by :

$$
p\left(\vec{x}_{1}, \ldots, \vec{x}_{s}\right)=\prod_{i=1}^{S} f_{i}\left(\vec{x}_{i}\right)
$$


The proof consists in showing that some detailed balance holds at every station (eq. 2-5) : it is a particular case of more general results in [Pel 1], [Pei 2] or [LeN] that also allow state-depending routing. Nevertheless, here is a direct proof :

Proof We start with some notations (due to PELLAUMAIL)

- $\mathrm{e}=\left(\mathrm{x}_{1}, \ldots, \mathrm{x}_{\mathrm{I}}\right)$ is a state of the network.

- $\tau_{k}\left(\hat{e}, s_{i}\right)$ (resp: $\tau_{S_{i}, k}\left(\overrightarrow{(}_{i}, s_{i}\right)$ ) is the left-hand side part of 2-3 when station $S_{i}$ is in network $R$ (resp : in isolation).

- $\tau_{k}\left(\stackrel{\uparrow}{e}, \stackrel{S}{S}_{i}\right)$ is the probability rate, in network $R$, of leaving state e through a transfer inside station $S_{i}$. We have self-comprehensive extensions to $\tau_{k, s_{i}}\left(\stackrel{\uparrow}{e}, \stackrel{o}{S_{i}}\right), \tau_{k}\left(\stackrel{\downarrow}{e}, \hat{s}_{i}\right) \ldots$

- we also note $\tau=\sum_{\text {class } k} \tau_{k} \quad$ for all kinds of $\tau_{s}^{\prime}$.

Now to prove the theorem, we show that, for all station $i$

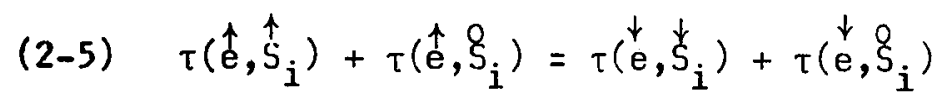

- on one side :

$L:=\tau\left(\hat{e}, \hat{S}_{i}\right)+\tau\left(\stackrel{\hat{e}, \hat{S}_{i}}{)}\right)=\frac{p(e)}{f_{i}\left(x_{i}\right)}\left[\tau_{S_{i}}\left(\hat{x}_{i}, \hat{S}_{i}\right)+\tau_{S_{i}}\left(\hat{x}_{i}, \stackrel{o}{S}_{i}\right)\right]$

- on the other :

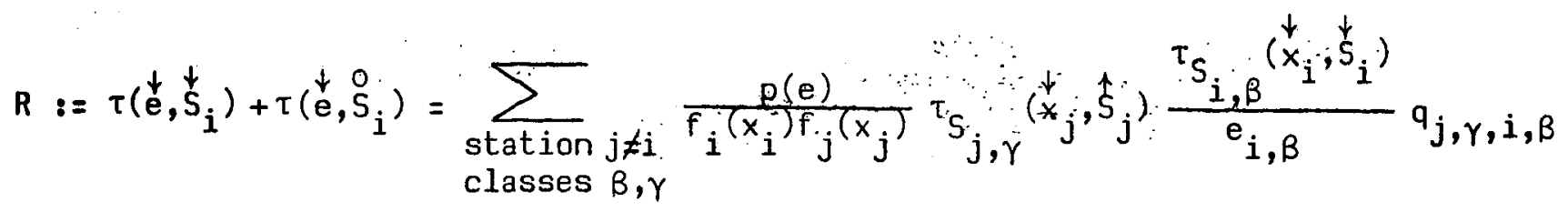

$$
\begin{aligned}
& +\sum_{\operatorname{classes} \beta} \frac{p(e)}{f_{i}\left(x_{i}\right)} \tau_{S_{i, \beta}}\left(\stackrel{\downarrow}{x}_{i}, \stackrel{s}{s}_{i}\right)
\end{aligned}
$$


now $\tau_{S_{j, \gamma}}\left(x_{j}, \stackrel{s}{s}_{j}\right)=f_{j}\left(x_{j}\right) e_{j, \beta}$ by local-balance property of station $s_{j}$ in isolation (eq. 2-3).

Hence we obtain :

$$
R:=\frac{p(e)}{f_{i}\left(x_{i}\right)} \sum_{\beta}\left[\tau_{S_{i, \beta}}\left(\stackrel{\downarrow}{x_{i}}, \stackrel{\downarrow}{S_{i}}\right)+\tau_{S_{i, \beta}}\left(\stackrel{\downarrow}{x_{i}}, \stackrel{o}{S}_{i}\right)\right]=\frac{p(e)}{f_{i}\left(x_{i}\right)}\left[\tau_{S_{i}}\left(\stackrel{\downarrow}{x_{i}}, \stackrel{\downarrow}{S_{i}}\right)+\tau_{S_{i}}\left(\stackrel{\downarrow}{x_{i}}, \stackrel{o}{S_{i}}\right)\right]
$$

Now (2-5) follows from (global) balance at station $S_{i}$ in isolation (eq. 2-4)

It is clear that summing (2-5) over all $\mathbf{i}$ gives balance equation. That ends the proof.

Remark 1 : Eq. 2-j does not generally hold by class. For instance, if $s_{i}$ is a FIFO station, $\tau_{k}\left(\hat{e}^{\uparrow}, \hat{S}_{i}\right)$ is zero if the customer receiving service does not belong to class $k$, whereas. $\tau_{k}\left({\stackrel{t}{e}, s_{i}}^{t}\right)$ is not necessarily zero.

Remark 2 : Local balance, as defined in 2-3, does not necessarily hold at every station of the product-form network (although it is true for every station in isolation). Actually, the proof of theorem 5 consists in showing that relations of type 2-4 hold at every station in the network.

It is known that BCMP networks are not locally balanced because of FIFO stations [S. C. ].

The same phenomenon occurs with concurrency multiserver stations; ve give an example :

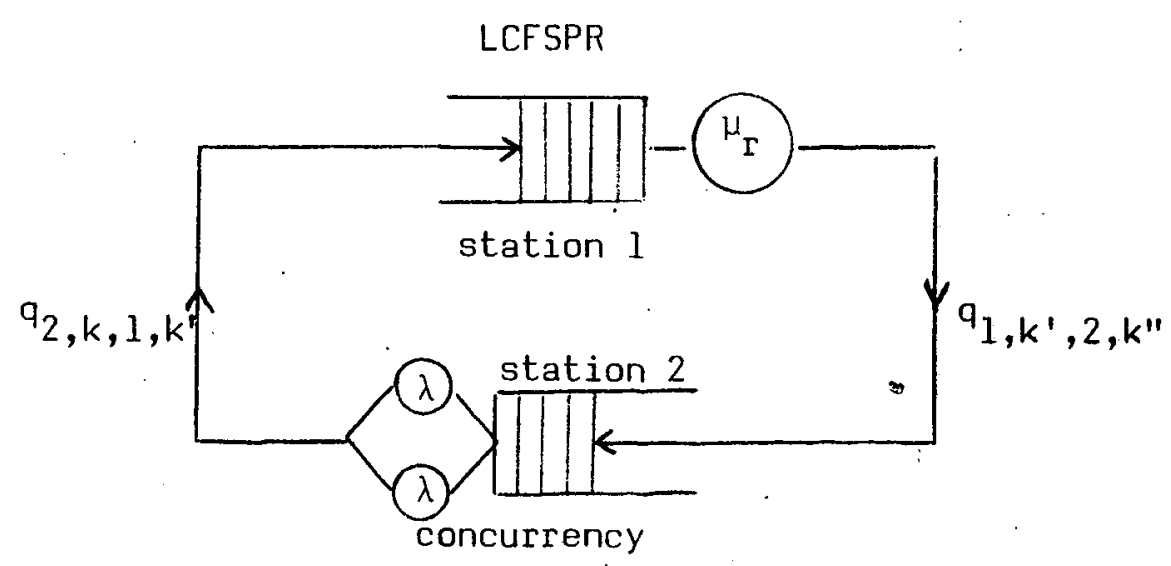


This is a multiclass network, in which customers may change classes according to probabilities $q_{i, c, j, c^{\prime}}$. A state of the network is $(\underline{x}, \underline{A}, \underline{c})$ where :

- $\underline{x}$ is the sequence of the classes of customers at station 1 , in last come first served order $\left(\underline{x}_{-}=\left(x_{1}, x_{2}, \ldots, x_{I}\right)\right)$

- A, $\underline{C}$ is a state of station 2 .

Rates of service are $\mu_{r}$ for class $r$ in station $1, \lambda$ in station 2 . The steady-state probability is given by :

$$
p(\underline{x}, A, \underline{c})=\prod_{i=1}^{I} \frac{e_{1, x_{i}}}{\mu_{x_{i}}} \times \underset{\gamma \in A}{\prod} \frac{e_{2, \gamma}}{\lambda} \times \prod_{j=1}^{j} \frac{e_{1, c_{j}}}{\lambda N_{A}(j)}
$$

The probability rate of leaving state $(\underline{x}, A, \underline{c})$ through an arrival of a class $k$ customer at station $l$ is :

(2-6) $\quad \tau_{k}\left((\underline{x}, \hat{A}, \underline{c}), \stackrel{\downarrow}{S_{1}}\right)=p(\underline{x}, A, \underline{c}) \times \sum_{l \in A} \lambda q_{2, l, l, k}$

while the dual rate is : (assuming the high level of station 2 is not empty)

$$
\tau\left((x, \stackrel{\downarrow}{A}, \underline{c}), \stackrel{\uparrow}{S_{1}}\right)=p\left(k+\underline{x}, A, \underline{c}-c_{\jmath}\right) \mu_{k} q_{1, k, 2, c_{j}}
$$

$$
\text { where }\left\{\begin{array}{l}
k+\underline{x}=\left(k, x_{1}, \ldots, x_{I}\right) \\
\underline{c-c_{J}}=\left(c_{1}, c_{2}, \ldots, c_{J-1}\right) \quad\left(\text { and } \underline{c}=\left(c_{1} \ldots c_{J}\right)\right)
\end{array}\right.
$$

hence :

$$
\tau_{k}\left((x, \stackrel{k}{A}, \underline{c}), \underline{S}_{1}\right)=p(\underline{x}, A, \underline{c}) e_{1, k} \times \lambda \frac{B}{e_{2, c_{j}}} q_{l, k, 2, c_{j}}
$$

Now $e_{1, k} \frac{B}{e_{2, c_{j}}} q_{1, k, 2, c_{j}}$ and $\sum_{\ell \in A} q_{2, l, l, k}$ are not generally equal, since the former depends on $c_{J}$ and the latter on $A$.

of course, when summing over $k$, we obtain 


$$
\sum_{k} e_{1, k} \frac{B}{e_{2, c_{j}}} q_{1, k, 2, c_{j}}=B \quad \text { and } \quad \sum_{l \in A} \sum_{k} q_{2, l, l, k}=B .
$$

\subsection{Aggregation of results}

We define aggregate states $\vec{y}_{i}$ and functions $g_{i}$ at each station :

* if station $i$ is a classical BCMP state, then :

$\vec{y}_{i}=\left(y_{i, 1} \cdots y_{i, c_{i}}\right)$ where $y_{i j}$ is the number of class $j$ customers at station $i ; c_{i}$ is the number of actual classes at station $i$,

(2-8) $g_{i}\left(\vec{y}_{i}\right)$ is the classical simplified function of BCMP station $i$, (as defined in [BCMP]) ; namely :

$g_{i}\left(\vec{y}_{i}\right)=\frac{y_{i} !}{\mu_{i}(1) \ldots \mu_{i}\left(y_{i}\right)} \prod_{\gamma=1}^{c_{i}} \frac{\left(e_{i, \gamma} v_{i, \gamma}\right)^{y} i, \gamma}{y_{i, \gamma} !}$

where $y_{i}=\left|\vec{y}_{i}\right|$ and $u_{i, \gamma}$ and $u_{i}\left(n_{i}\right)$ depend on the type of the station :

- $w_{i, \gamma}=\frac{\mu_{i}}{\mu_{i, \gamma}}$ and $\mu_{i}\left(n_{i}\right)=\mu_{i}$ at PS or LCFSPR stations

$-w_{i, \gamma}=\frac{\mu_{i}}{\mu_{i, \gamma}}$ and $\mu_{i}\left(n_{i}\right)=\mu_{i} \times n_{i}$ at Is stations

$-w_{i, \gamma}=1$ and $\mu_{i}\left(n_{i}\right)=\mu_{i}$ at FCFS stations

[Pit]

* if station $i$ is a concurrency multiserver station, then :

$\vec{y}=\left(A c, \vec{n}_{1}, \ldots, \vec{n}_{M_{i}}\right)$ where :

- Ac is the set of active classes at station $i$

- $\vec{n}_{m}=\left(n_{m, 1}, \ldots, n_{m, l_{m}}\right)$ where $n_{m, \gamma}$ is the number of class $(m, \gamma)$-customers at station $i$. 
we also define :

- $\vec{n}=\left(\left|\vec{n}_{1}\right|, \ldots,\left|\vec{n}_{M_{i}}\right|\right)$, the vector whose $m^{\text {th }}$ component is the number of group-m customers

- $A g=$ the set of active groups : if $A c=\left\{\left(m_{1}, k_{1}\right) \ldots\left(m_{R}, k_{\Gamma}\right)\right\}$ then $A g=\left\{m_{1}, \ldots, m_{R}\right\}$

- and function $g_{i}$

(2-9) $g_{i}\left(A c, \vec{n}_{1}, \ldots, \vec{n}_{M_{i}}\right)=\prod_{\gamma \in A c} \rho_{\gamma} \prod_{m=1}^{M_{i}}\left(\frac{\left|\vec{n}_{m}\right| !}{\vec{n}_{m} \mid} \prod_{k=1}^{c_{m}} \frac{\rho_{m, k}}{\vec{n}_{m, k} !}\right) \times \pi_{A g}(\vec{n})$ where $\pi_{A g}(\vec{n})$ is recursively defined by :

$$
\left\{\begin{array}{l}
\pi_{A g}(\vec{n})=B^{|\vec{n}|} \text { if } n_{m}=0 \text { for all } m \text { not in } A g \\
\pi_{A g}(\vec{n})=\sum_{m / n_{m}>0} \pi_{A g}\left(\vec{n}_{m^{-}}\right) \text {else }
\end{array}\right.
$$

Then, under the assumptions of theorem 5, we have :

$$
\begin{array}{ll}
\text { Corollary } & \text { The steady-state probabilities of the aggregated states are : } \\
(2-10) & P\left(\vec{y}_{1}, \ldots, \vec{y}_{S}\right)=k \prod_{i=1}^{S} g_{i}\left(\vec{y}_{i}\right) \\
& \text { where } g_{i} \text { are defined in }(2-8) \text { and }(2-9) .
\end{array}
$$

Proof : see section 1-4 for a detailed proof in the "concurrency multiserver" case.

Remark : Aggregation formulas (2-10) hold only if the restrictions about feasible states are due to the total number of customers in the system. 
CONCLUSION

The range of product-form networks has been extended to models for which mutual exclusion is permitted.

Aknowledgement

I would like to thank Jean PELLAUMAIL and Louis Marie LE NY for fruitful discussions about the topic of section 2 . 
APPENDIX : Proof of the lemmas

Lemma $1 \quad \sum_{b \in A} \sum_{l=0}^{D(b)} \quad \frac{1}{B} \prod_{\ell^{\prime}=\ell+1}^{S} \frac{N\left(\ell^{\prime}\right)}{B}=1$

the assumptions are these of $1-3-b), 1$ st case ; call :

- $n_{z}$ the number of positions belonging to level $z$.

- $Z(b)=N(D(b))$ the last lower level with no class-b-customer.

- L the last level.

then :

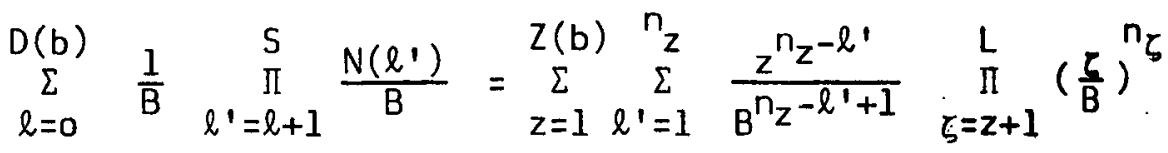

$$
\begin{aligned}
& +\frac{1}{B} \prod_{\zeta=1}^{L}\left(\frac{\zeta}{B}\right)^{n_{\zeta}} \\
& =\sum_{z=0}^{Z(b)} \frac{i-\left(\frac{z}{B}\right)^{n}}{B-n_{z}} \sum_{\zeta=z+1}^{L}\left(\frac{\zeta}{B}\right)^{n_{\zeta}}
\end{aligned}
$$

(with our convention : $n_{0}=0$ )

Calling MI the left-hand-side part of lemma 1, we obtain :

$$
M I=\sum_{b \in A} \sum_{z=0}^{Z(b)} \frac{1-\left(\frac{z}{B}\right)^{n_{z}}}{B-z} \prod_{\zeta=z+1}^{L}\left(\frac{\zeta}{B}\right)^{n_{\zeta}}
$$

now $" z \leqslant Z(b) "$ means that there is no class $b$ customer in levels 1 to $z$ of the waiting room ; but for a given level, $z$, there are exactly $\mathrm{B}-\mathrm{z}$ such classes (we have assumed here that $\mathrm{b}$ is an active class, and that all servers are busy). That still holds for $z=0$.

Hence we can write : 


$$
M 1=\sum_{z=0}^{L^{\prime}}\left(1-\left(\frac{z}{B}\right)^{n_{z}}\right) \prod_{\zeta=z+1}^{L}\left(\frac{\zeta}{B}\right)^{n_{\zeta}}
$$

where $L^{\prime}$ is the last lower level :

if $L=B$ then $L^{\prime}=L-1$ else $L^{\prime}=L$.

Thus $\prod_{\zeta=z+1}^{L}\left(\frac{\zeta}{B}\right)^{n_{\zeta}}=\prod_{\zeta=z+1}^{L^{\prime}}\left(\frac{\zeta}{B}\right)^{n_{\zeta}}$ and we obtain :

$$
M I=\sum_{z=0}^{L^{\prime}}\left(u_{z+1}-u_{z}\right) \quad \text { with } u_{z}=\prod_{\zeta=z}^{L^{\prime}}\left(\frac{\zeta}{B}\right)^{n_{\zeta}}, u_{L^{\prime}+1}=1, u_{0}=0
$$

That ends the proof of lemma 1 .

Lemma 2 $\quad$\begin{tabular}{cccc|}
$\sum_{0 \leqslant \ell \leqslant D(k)}$ & $\frac{1}{N(\ell)+1}$ & $\prod_{\ell^{\prime}=\ell+1}$ & $\frac{N\left(\ell^{\prime}\right)}{N\left(\ell^{\prime}\right)+1}=1$ \\
\hline
\end{tabular}

Now the assumptions are those of section 1-3-b), 3rd case, and we call M2 the left-hand-side of the lemma. We keep the same notations as in lemma 1.

We have :

$$
M 2=\sum_{z=1}^{Z(k)} \sum_{l=1}^{n_{z}} \frac{1}{z+1}\left(\frac{z}{z+1}\right)^{n_{z}-l} \prod_{\zeta=z+1}^{Z(k)}\left(\frac{\zeta}{\zeta+1}\right)^{n_{\zeta}}+\prod_{\zeta=1}^{D(k)}\left(\frac{\zeta}{\zeta+1}\right)^{n_{\zeta}}
$$

Hence

$$
M 2=\sum_{z=0}^{Z(k)}\left(1-\left(\frac{z}{z+1}\right)^{n_{z}}\right) \prod_{\zeta=z+1}^{Z(k)}\left(\frac{\zeta}{\zeta+1}\right)^{n_{\zeta}}
$$

Now the end of the proof uses the same manipulation as in lemma 1. 


\section{REFERENCES}

[BCDM] BALBO, CHIOLA, DONATELLI, MARSAN, "On the product form solution of a class of multiple bus multiprocessor system models", Proc. of the int. workshop on modeling and performance evaluation of parallel systems, Grenoble 1984.

[BCMP] BASKETT, CHANDY, MUNTZ and PALACIOS, "Open, closed and mixed networks of queues with different classes of customers", J.A.C.M. vol. $22, n^{0} 2,248-260,1975$.

[CHT] CHANDY, HOWARD and TOWSLEY, "Product form and local balance in queueing networks", Journal of the ACM, vol. 24, $n^{\circ} 2,1977$.

[GeP] GELENBE et PUJOLLE, "Introduction aux réseaux de files d'attente", Eyrolles, 1982.

[Jac] JACKSON, "Jobshop-like queue system", Management Sci., vol. 10, 131-142, 1963.

[LeN] LE NY, "Etude analytique de réseaux de files d'attente multiclasses à routages variables", RAIRO, vol. $14, n^{0} 4,331-347,1980$.

[Mun] MUNTZ, "Poisson departure processes and queueing networks", IBM Res. Rep. RC-4145, 1972.

[Pell] PELLAUMAIL, "Formule du produit et décomposition de réseaux de files d'attente", Ann. Inst. Henri Poincaré, vol. 15, n³ , 261-286, 1979.

[Pel2] PELLAUMAIL, "Solutions à forme produit pour des systèmes linéaires", rapport IRISA, $n^{0}, 1984$.

[Pit] PITTEL, "Closed exponential networks of queues with blocking", I.B.M. Research report, RC. 7174, 1983.

[SC] SAUER, CHANDY, "Computer system performance modeling", Prentice Hall, 1981. 
PI 238 Algorithme optimal de décision pour l'équivalence des grammaires simples

Didier Caucal, 48 pages ; Septembre 1984.

PI 239 Detection and diagnosis of abrupt changes in modal characteristics of nonstationary digital signals

Michèle Basseville. Albert Benveniste. Georges Moustakides, 26 pages : Octobre 1984.

PI 240 Convergence optimale de l'algorithme de "réallocation-recentrage" dans le cas le plus pur

Israël-César Lerman. 39 pages : Octobre 1984.

PI 241 Un algorithme d'exclusion mutuelle pour une structure logique en anneau

Michel Raynal. 12 pages : Novembre 1984.

PI 242 Note sur l'interprétation de primitives d'action proximétriques en termes de liaisons cinématiques fictives

Bernard Espiau, 20 pages : Novembre 1984

PI 243 Robust detection of signals - A large deviations approach

Georges V. Moustakides. 16 pages : Novembre 1984.

PI 244 Algorithmes de génération de caractères

Gerard Hégron. 24 pages: Novembre 1984

PI 245 Optimal stopping times for detecting changes in distributions

Georges V. Moustakides. 10 pages : Janvier 1985.

P1 246 Signal : a data flow oriented language for signal processing

Paul Le Guernic. Albert Benveniste. Patricia Bournai. Thierry Gautier. 62 pages: Janvier 1985 .

PI 247 On syntax and semantics of adjective phrases in logic programming Patrick Saint-Dizier. 20 pages : Fevrier 1985.

PI 248 Modélisation des robots manipulateurs rigides Nichel Le Borgne. 75 pages: Février 1985.

Pl $2+9$ Detecting changes in the A.R. parameters of a nonstationary A.R.M.A. process

Georges 1. Moustakides. Albert Benveniste. 22 pages; Mars 1985.

PI 250 A BCMP extension to multiserver stations with concurrent classes of customers

Jean-Y'ves Le Boudec. 32 pages : Mars 1985.

PI 251 An approach to natural language semantics in logic programming Patrick Saint-Dizier. 34 pages : Mars 1985. 
$\therefore$ 